\title{
LASER-INDUCED THERMAL DESORPTION OF CO ON Ni(111): DETERMINATION OF PRE-EXPONENTIAL FACTOR AND HEAT OF DESORPTION
}

\author{
X.D. ZHU, Th. RASING and Y.R. SHEN \\ Department of Physics, University of California, \\ and Materials and Chemical Sciences Division, Lawrence Berkeley Laboratory, Berkeley, CA 94720, USA
}

Received 18 October 1988; in final form 22 December 1988

\begin{abstract}
We show that by combining laser-induced thermal desorption with conventional thermal desorption spectroscopy, one can determine separately the activation energy $E_{\mathrm{d}}$ and the pre-exponential factor $\nu$ for desorption with good accuracy. Results on CO on $\mathrm{Ni}(111)$ yield $E_{\mathrm{d}} \approx 25.5 \mathrm{kcal} / \mathrm{mol}$ and $\nu \approx 10^{13} / \mathrm{s}$. They agree well with values obtained by other techniques and suggest that above room temperature, $\mathrm{CO}$ on $\mathrm{Ni}(111)$ is in a highly mobile gas-like phase.
\end{abstract}

Molecular adsorption and desorption are subjects of great importance in modern surface science as they provide information on the binding energy of adsorbates, states of adsorption, and nature of adsorbate-substrate and adsorbate-adsorbate interactions [1]. Desorption is governed by the activation energy $E_{\mathrm{d}}$ and the pre-exponential factor $\nu$. The former describes the energy required for a molecule to desorb, while the latter is related to the state (mobile or immobile) the adsorbates are in prior to desorption [2,3]. Experimental determination of $E_{\mathrm{d}}$ and $\nu$ and their dependences on the surface coverage in comparison with theory will therefore allow a better understanding of an adsorbate-substrate system. Conventional thermal desorption spectroscopy (TDS) is often used to deduce $E_{\mathrm{d}}$ and $\nu$. It has, however, been recognized that a separate determination of $E_{\mathrm{d}}$ and $\nu$ with good accuracy requires a very careful execution of the experiment. Measurements of the number of desorbed molecules must be highly accurate and free of background noise [2-6]. Several methods have been developed to bypass the difficulties [7-11]. In particular, it has been pointed out that a set of TDS measurements with very different heating rates should facilitate the unambiguous determination of $E_{\mathrm{d}}$ and $\nu$ [8,9]. Unfortunately, the heating rate of a substrate is often limited by the heating method.

With the help of high-power pulsed lasers, it is now possible to heat a surface with a heating rate larger than $10^{9} \mathrm{~K} / \mathrm{s}$ [12-14]. This has been utilized by Hall [15] to observe thermally induced surface reactions characteristically different from those induced by slow heating. In this Letter, we show that pulsed laser heating to induce thermal desorption in combination with a conventional TDS (with a heating rate of $\approx 1 \mathrm{~K} / \mathrm{s}$ ) also offers a relatively simple method to determine $E_{\mathrm{d}}$ and $\nu$ fairly accurately. To measure the changes in the surface coverage $\theta$ due to laser desorption, we used optical second-harmonic generation (SHG) as an in situ probe. This avoids complication of the dependence of the signal on the beam profile that may arise when mass spectrometry is used to monitor the desorbed molecules [15]. We took $\mathrm{CO}$ on $\mathrm{Ni}(111)$ as a model system to try out the method, and found $E_{\mathrm{d}} \approx 25.5 \mathrm{kcal} / \mathrm{mol}$ and $\nu \approx 10^{13} /$ $\mathrm{s}$ in the range of $0.1<\theta<0.9$, in good agreement with those obtained by other techniques $[5,10]$.

The desorption rate of molecules from a surface can be described by $[2,3]$

$\mathrm{d} \theta / \mathrm{d} t=\nu \theta \exp \left(-E_{\mathrm{d}} / R T\right)$.

Conventional TDS studies the change in coverage with temperature $\mathrm{d} \theta / \mathrm{d} T=(\mathrm{d} \theta / \mathrm{d} t) /(\mathrm{d} T / \mathrm{d} t)$ by observing the mass yield while heating up the substrate by a prescribed heating rate $\mathrm{d} T / \mathrm{d} t$. With $\mathrm{SHG}$, the surface coverage $\theta(T)$ (normalized against the sat- 
uration coverage), can be directly monitored [16]. From eq. (1), one finds

$\theta(T)=\exp \left(-\int_{T_{0}}^{T(t)} \frac{\nu}{\mathrm{d} T / \mathrm{d} t} \exp \left(-E_{\mathrm{d}} / R T\right) \mathrm{d} T\right)$

where $T_{0}$ is the initial temperature.

According to statistical theories $[2,6]$ of molecular desorption, the pre-exponential factor $\nu$ depends critically on the state the adsorbates are in. If the adsorbates are free to move around on the surface, $\nu$ is roughly given by $\nu \approx k_{\mathrm{B}} T / h \approx 10^{13} / \mathrm{s}$. On the other hand, if the adsorbates are immobile on the surface, the relatively large entropy difference between the vapor and the adsorbed phases leads to an increase of $\nu$ by $\approx 10^{3}$, that is, $\nu \approx 10^{16} / \mathrm{s}$. Experimentally due to relatively low heating rates in conventional TDS $(1-10 \mathrm{~K} / \mathrm{s})$, one would find from eq. (1) or (2) that unless the TDS data have unusually high accuracy, it is rather difficult to deduce $E_{\mathrm{d}}$ and $\nu$ scparatcly from the results [2-6]. This is illustrated in fig. 1. For $\mathrm{d} T / \mathrm{d} t=1 \mathrm{~K} / \mathrm{s}$, the two TDS curves of $\Delta \theta(T)$ $(=1-\theta(T))$ that are calculated from eq. (2) with $E_{\mathrm{d}}=25.5 \mathrm{kcal} / \mathrm{mol}, \nu=10^{13} / \mathrm{s}$ and $E_{\mathrm{d}}=32.9 \mathrm{kcal} /$ mol, $\nu=10^{16} / \mathrm{s}$, respectively, can hardly be resolved. If, however, the heating rate could be increased to, say, $\mathrm{d} T / \mathrm{d} t=10^{9} \mathrm{~K} / \mathrm{s}$, then the two curves with the same sets of values of $E_{\mathrm{d}}$ and $\nu$ are widely separated, as is also depicted in fig. 1 . We should note that it is also difficult to separately determine $\nu$ and $E_{\mathrm{d}}$ solely from thermal desorption with a rapid heating rate.

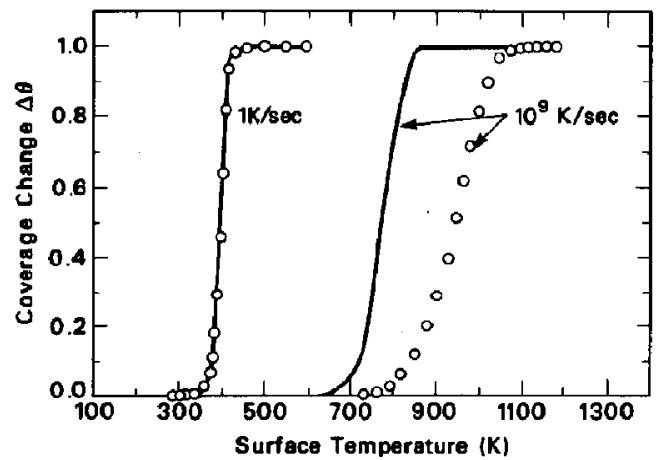

Fig. 1. Calculated thermal desorption spectra for heating rates of 1 and $10^{9} \mathrm{~K} / \mathrm{s}$. The solid line corresponds to $\nu \approx 10^{16} / \mathrm{s}$ and $E_{\mathrm{d}} \approx 32.9 \mathrm{kcal} / \mathrm{mol}$. The open circles correspond to $\nu \approx 10^{13} / \mathrm{s}$ and $E_{\mathrm{d}} \approx 25.5 \mathrm{kcal} / \mathrm{mol}$. At $\mathrm{d} T / \mathrm{d} t=1 \mathrm{~K} / \mathrm{s}$, the two curves overlap.
For example, for $\mathrm{d} T / \mathrm{d} t=10^{9} \mathrm{~K} / \mathrm{s}$, the TDS curve with $E_{\mathrm{d}}=25.5 \mathrm{kcal} / \mathrm{mol}$ and $\nu=10^{13} / \mathrm{s}$ can hardly be resolved from that with $E_{\mathrm{d}}=40.1 \mathrm{kcal} / \mathrm{mol}$ and $\nu=10^{16} / \mathrm{s}$. Thus it is obvious that one should like to use TDS with two vastly different heating rates in order to deduce $\nu$ and $E_{\mathrm{d}}$ separately and accurately.

As mentioned before, the heating rate in conventional TDS is usually limited. Pulsed laser heating, however, can easily yield a rate larger than $10^{\circ} \mathrm{K} / \mathrm{s}$ [15]. Instantaneous local thermal equilibrium can be expected for nanosecond pulsed laser heating of a metal surface since electron-electron and electronphonon relaxation times in a metal are in the picosecond and subpicosecond range. Although one finds it difficult to measure the coverage as a function of the temperature when the latter changes at a rate of $10^{9} \mathrm{~K} / \mathrm{s}$, we realize that the same goal can be achieved equally well by measuring $\theta(t \rightarrow \infty)$ as a function of the pulsed laser energy. Theoretically, knowing the laser energy adsorbed, the temporal and spatial profiles of the laser pulse, and the thermal properties of the substrate, we can calculate the local temperature variation of the surface with time using, for example, the equations of Bechtel [17] $\# 1$. We believe that with the laser flux known to within $4 \%$, the prediction of a temperature rise can be better than $\pm 40 \mathrm{~K}$. The resultant overall thermal desorption due to the pulse heating can then be found following eq. (2). In fig. 2 , we show the two clearly resolved theoretical curves describing the overall laser-induced thermal desorption as a function of the laser fluence for $\mathrm{CO}$ on $\mathrm{Ni}(111)$ with the same two sets of parameters $E_{\mathrm{d}}$ and $\nu$ assumed in fig. 1. The laser pulse incident on the sample was assumed to have a temporal profile of $I=I_{0} \exp \left(-t^{2} / \tau_{\mathrm{p}}^{2}\right)$ with $\tau_{\mathrm{p}}=10 \mathrm{~ns}$ and a spatial profile of $I=I_{0} \exp \left(-r^{2} / a^{2}\right)$ with $a=0.25 \mathrm{~cm}$. They were chosen to simulate the actual laser pulse used in our experiment. The calculation refers to desorption from the center of the beam spot on the sample over which the temperature is uniform.

The experiment was carried out in a UHV cham-

\footnotetext{
\#1 For optical properties of a $\mathrm{Ni}$ single crystal, we have used the updated results, $n(\lambda=1.064 \mu \mathrm{m})=2.91+\mathrm{i} 5.24$, published in ref. [18]. We measured the reflectivity of the $\mathrm{Ni}$ and found it the same from room temperature to $1100 \mathrm{~K}$ over which the substantial desorption of $\mathrm{CO}$ takes place. For thermal properties of a $\mathrm{Ni}$ single crystal, we have adopted the values of heat capacity and thermal conductivity of $\mathrm{Ni}$ published in ref. [19].
} 


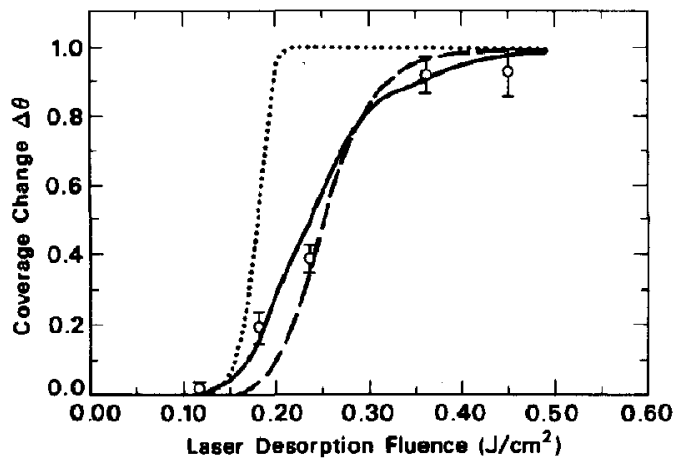

Fig. 2. Experimental laser-induced thermal desorption (LITD) data (open circles), together with theoretical predictions. Dotted line: $\nu \approx 10^{16} / \mathrm{s}$ and $E_{\mathrm{d}} \approx 32.9 \mathrm{kcal} / \mathrm{mol}$. Dashed line: $\nu \approx$ $10^{13} / \mathrm{s}$ and $E_{\mathrm{d}}=25.5 \mathrm{kcal} / \mathrm{mol}$. Solid curve corresponds to $\nu=$ $0.91\left(k_{\mathrm{B}} T / h\right) \theta$ and $E_{\mathrm{d}}=E_{\mathrm{d}}(\theta)-\frac{3}{2} R T$ taken from ref. [9].

ber equipped with the usual cleaning and analyzing tools. The operating pressure was maintained below $(1-2) \times 10^{-10}$ Torr. The Ni(111) sample, $99.995 \%$ pure, was mechanically polished (with $0.05 \mu \mathrm{m} \mathrm{Al} \mathrm{O}_{3}$ powder) and afterwards annealed at $1000 \mathrm{~K}$ in a hydrogen atmosphere for a week to remove bulk sulfur. The sample was then routinely cleaned with $5 \times 10^{-5}$ Torr $\mathrm{Ar}^{+}$sputtering with a $500 \mathrm{~V}$ beam voltage at $550^{\circ} \mathrm{C}$ for $2 \mathrm{~h}$ and followed by annealing during the slow cooling down to room temperature. The clean sample surface showed hardly any trace of $\mathrm{C}$ and $\mathrm{O}$ and less than $0.5 \%$ of a monolayer of $S$ as checked by Auger spectroscopy. Sharp and clean $(1 \times 1)$ LEED patterns indicated a well-ordered surface layer. The sample was additionally flash heated to $620 \mathrm{~K}$ right before each experimental run. All the measurements were carried out at an initial sample temperature of $273 \mathrm{~K}$.

Conventional TDS spectra were obtained with $d T$ / $\mathrm{d} t=2 \mathrm{~K} / \mathrm{s}$, showing a desorption maximum that shifts from $425 \mathrm{~K}$ at low coverages to $408 \mathrm{~K}$ at the saturation coverage, in agreement with earlier measurements $[5,20]$. For the laser-induced desorption we employed the output of a single-mode $Q$-switched $\mathrm{Nd}$ : YAG laser at $1.064 \mu \mathrm{m}$ with the spatial and temporal profiles mentioned earlier. To probe the surface coverage of $\mathrm{CO}$ remaining on $\mathrm{Ni}(111)$ after laser desorption, we used surface SHG with the frequency-doubled output at $0.532 \mu \mathrm{m}$ of the same laser source as the pump beam. The effectiveness of SHG as a surface probe has been demonstrated recently [16] and stems from the fact that in the electric dipole approximation the SHG process is forbidden in centrosymmetric media but necessarily allowed at symmetry breaking interfaces. Due to the high polarizability of the nearly free electrons, metal surfaces usually exhibit a high nonlinearity and thus yield a large SHG signal. The presence of $\mathrm{CO}$ adsorbates will greatly reduce this surface nonlinearity and therefore the observed SHG signal will be very sensitive to the surface coverage. By calibrating SHG against TDS we can use the optical technique to measure the absolute coverage to within a few percent [16]. The probe beam was focused to a spot 30 times smaller than the desorbing beam on the sample. Thus only the central part of the desorbed area was monitored. Both laser beams came in collinearly at an angle of $40^{\circ}$ from the surface normal. For desorption, the deposited energy from a single pulse was varied from 0.12 to $0.45 \mathrm{~J} / \mathrm{cm}^{2}$ to within $4 \%$. The incident probe beam had a fluence of $0.07 \mathrm{~J} / \mathrm{cm}^{2}$ per pulse at a $10 \mathrm{~Hz}$ repetition rate. During the time lapse of a few minutes between pumping and probing, surface diffusion and readsorption were found to be negligible [14].

The observed change in the CO surface coverage due to laser-induced desorption as a function of laser fluence is plotted in fig. 2 , in comparison with the theoretical curves. It shows that the experiment strongly favors the choice of $\nu \approx 10^{13} / \mathrm{s}$ and $E_{\mathrm{d}}=25.5$ $\mathrm{kcal} / \mathrm{mol}$ (dashed line) which agrees with the conclusion of the recent EELS [10] and TDS [5] studies on the same system. Uncertainty of $\pm 40 \mathrm{~K}$ in the temperature variation in the theoretical calculation would not affect the values of $\log \nu$ and $E_{\mathrm{d}}$ by more than $5 \%$. For small $\Delta \theta$, there is some discrepancy between theory and experiment which appears to be due to our assumption of a coverage-independent $E_{\mathrm{d}}$ and $\nu$. Isosteric heat of adsorption measurements of $\mathrm{CO}$ on $\mathrm{Ni}(111)$ have shown a coverage dependence of $E_{\mathrm{d}}$ [21]. Using those data for $E_{\mathrm{d}}(\theta)$ and $\nu=0.91\left(k_{\mathrm{B}} T / h\right) \theta$ derived from the statistical theory that assumes uninhibited motion of $\mathrm{CO}$ on the surface $\# 2$, we obtained a theoretical curve which agrees well with our experimental results, as is shown

\footnotetext{
\#2 See, for example, ref. [2]. A more complete statistical treatment will be published separately.
} 
by the solid line in fig. 2. (The dependence of $\nu$ on $T$ and $\theta$ is not very significant. In comparison with the $\Delta \theta$ curve calculated by assuming $\nu=10^{13} / \mathrm{s}$, the difference is only appreciable as $\Delta \theta$ approaches 1.0 .)

As discussed by Ibach et al. [6], our result of $\nu \approx 10^{13} / \mathrm{s}$ indicates that above room temperature, $\mathrm{CO}$ on $\mathrm{Ni}(111)$ moves more or less freely over the surface, and is not bound to a particular site during most of its residence time. This is in agreement with the results of the TDS and EELS studies $[5,10]$. The latter yielded $\nu=3 \times 10^{12} / \mathrm{s}$ (within one order of magnitude) and found that the desorption rates of $\mathrm{CO}$ from the top and bridge sites are practically the same at such low coverages as $\theta \approx 10^{-3}-0.4$. Stochastic trajectory simulations used to study NO on $\mathrm{Ag}$ (111) and $\mathrm{Pt} \mathrm{(111)} \mathrm{seemed} \mathrm{to} \mathrm{have} \mathrm{suggested} \mathrm{that}$ diatomic molecules adsorbed on metal surfaces would have their lateral motion nearly frozen even at fairly high temperatures [22]. This would lead to $\nu \approx 10^{16} / \mathrm{s}$, which seems to be the case for $\mathrm{CO}$ on $\mathrm{Ru}(100)$ [9]. Our result here shows that for $\mathrm{CO}$ on $\mathrm{Ni}(111)$, the opposite is true.

In conclusion, we have demonstrated that by combining the conventional thermal desorption spectroscopy with pulsed laser-induced thermal desorption measurements, and using optical secondharmonic generation as an in situ probe for the surface coverage, we can determine the desorption kinetics parameters quite accurately. We found that for $\mathrm{CO}$ on $\mathrm{Ni}(111)$, the desorption of $\mathrm{CO}$ is characterized by a pre-exponential factor of $\nu \approx 10^{13} / \mathrm{s}$ and an average heat of adsorption $E_{\mathrm{d}} \approx 25.5 \mathrm{kcal} / \mathrm{mol}$. The latter changes from $21 \mathrm{kcal} / \mathrm{mol}$ at $\theta=0.9$ to $27 \mathrm{kcal} /$ $\mathrm{mol}$ at $\theta=0.1$. The result indicates that $\mathrm{CO}$ on $\mathrm{Ni}(111)$ is in a highly mobile gas-like phase.

This work was supported by the Director, Office of Energy Research, Office of Basic Energy Sciences, Materials Sciences Division of the US Department of Energy under Contract No. DE-AC03-76SF00098.

\section{References}

[1] G.A. Somorjai, Chemistry in two dimensions: surfaces (Cornell Univ. Press, Ithaca, 1981 ) p. 360.

[2] M.A. Morris, M. Bowker and D.A. King, in: Chemical kinetics, Vol. 19, eds. C.H. Bamford, C.F.H. Tipper and R.G. Compton (Elsevier, Amsterdam, 1984) pp. 1-163.

[3] D.A. King, Surface Sci. 47 (1983) 384.

[4] J.M. Soler and N. Garcia, Surface Sci. 124 (1983) 563.

[5] J.B. Miller, H.R. Siddiqui, S.M. Gates, J.N. Russell Jr., J.T. Yates Jr, J.C. Tully and M.J. Cardillo, J. Chem. Phys. 87 (1988) 6725 .

[6] H. Ibach, W. Erley and H. Wagner, Surface Sci. 92 (1980) 29 , and references therein.

[7] D.A. King, T.E. Madey and J.T. Yates Jr., J. Chem. Phys. 55 (1971) 3236.

[8] J.L. Taylor and W.H. Weiberg, Surface Sci. 78 (1978) 259.

[9] H. Pfnur, P. Feulner, H.A. Engelhardt and D. Menzel, Chem. Phys. Letters 59 (1978) 481.

[10] H. Froitzheim, U, Kohler and H. Lammering, Phys. Rev. B 34 (1986) 2125.

[11] E. Habenschaden and J. Küppers, Surface Sci. 138 (1984) L147.

[12] R. Viswanathan, D.R. Burgess Jr., P.C. Stair and E. Weitz, J. Vacuum. Sci. Technol. 20 (1982) 605;

S.M. George, A.M. DeSantolo and R.B. Hall, Surface Sci. 159 (1985) L425.

[13] E.G. Seebauer, A.C.F. Kong and L.D. Schmidt, J. Chem. Phys. 88 (1988) 6597, and references therein.

[14] X.D. Zhu, Th. Rasing and Y.R. Shen, Phys. Rev. Letters, submitted for publication.

[15] R.B. Hall, J. Phys. Chem. 91 (1987) 1007.

[16] X.D. Zhu, Y.R. Shen and R. Carr, Surface Sci. 163 (1985) $114 ;$

H.W.K. Tom, C.M. Mate, X.D. Zhu, J.E. Crowell, T.F. Heinz, G.A. Somorjai and Y,R, Shen, Phys. Rev, Letters 52 (1984) 348;

H.W.K. Tom, C.M. Mate, X.D. Zhu, J.E. Crowell, Y.R. Shen and G.A. Somorjai, Surface Sci. 172 (1986) 466, and references therein;

S.G. Grubb, A.M. DeSantolo and R.B. Hall, J. Phys. Chem. 92 (1988) 1419.

[17] J.H. Bechtel, J. Appl. Phys. 46 (1975) 1585.

[18] J.H. Weaver, C. Krafka, D.W. Lynch and E.E. Koch, Physics data, No. 18-1. Optical properties of metals (FachInformations-Zentrum Energie, Physik, Mathematik GmbH, Karlsruhe, 1981 ) pp. 95-108.

[19] D.E. Gray, ed., American Institute of Physics handbook, 3rd Ed. (McGraw-Hill, New York, 1972) pp. 4-106, 4-154, and references therein.

[20] F.P. Netzer and T.E. Madey, J. Chem. Phys. 76 (1982) 710, and references therein.

[21 ] K. Christmann, O. Schöber and G. Ertl, J. Chem. Phys. 60 (1974) 4719.

[22] C.W. Muhlhausen, L.R. Williams and J.C. Tully, J. Chem. Phys. 83 (1985) 2594. 